\title{
Fundamental Harmonic Power Laws Relating the Frequency Equivalents of the Electron, Bohr Radius, Rydberg Constant with the Fine Structure, Planck's Constant, 2 and $\pi$
}

\author{
Donald William Chakeres \\ Department of Radiology, The Ohio State University, Columbus, OH, USA \\ Email: donald.chakeres@osumc.edu
}

How to cite this paper: Chakeres, D.W. (2016) Fundamental Harmonic Power Laws Relating the Frequency Equivalents of the Electron, Bohr Radius, Rydberg Constant with the Fine Structure, Planck's Constant, 2 and $\pi$. Journal of Modern Physics, 7, $1801-1810$

http://dx.doi.org/10.4236/jmp.2016.713160

Received: August 3, 2016

Accepted: September 27, 2016

Published: September 30, 2016

Copyright $\odot 2016$ by author and Scientific Research Publishing Inc. This work is licensed under the Creative Commons Attribution International License (CC BY 4.0).

http://creativecommons.org/licenses/by/4.0/

(c) (i) Open Access

\begin{abstract}
We evaluate three of the quantum constants of hydrogen, the electron, $e^{-}$, the Bohr radius, $a_{0}$, and the Rydberg constants, $R_{\infty}$, as natural unit frequency equivalents, $v$. This is equivalent to Planck's constant, $h$, the speed of light, $c$, and the electron charge, $e$, all scaled to 1 similar in concept to the Hartree atomic, and Planck units. These frequency ratios are analyzed as fundamental coupling constants. We recognize that the ratio of the product of $8 \pi^{2}$, the $V_{e-}$ times the $V_{R}$ divided by $V_{a 0}$ squared equals 1 . This is a power law defining Planck's constant in a dimensionless domain as 1. We also find that all of the possible dimensionless and dimensioned ratios correspond to other constants or classic relationships, and are systematically inter-related by multiple power laws to the fine structure constant, $\alpha$; and the geometric factors 2 , and $\pi$. One is related to an angular momentum scaled by Planck's constant, and another is the kinetic energy law. There are harmonic sinusoidal relationships based on $2 \pi$ circle geometry. In the dimensionless domain, $\alpha$ is equivalent to the free space constant of permeability, and its reciprocal to permittivity. If any two quanta are known, all of the others can be derived within power laws. This demonstrates that $8 \pi^{2}$ represents the logical geometric conversion factor that links the Euclid geometric factors/three dimensional space, and the quantum domain. We conclude that the relative scale and organization of many of the fundamental constants even beyond hydrogen are related to a unified power law system defined by only three physical quanta of $v_{e^{-}}, v_{R}$, and $v_{a_{0}}$.
\end{abstract}

\section{Keywords}

Fundamental Physical Constants, Unification Models, Hydrogen, Electron, Bohr Radius, Rydberg Constant, Fine Structure Constant 


\section{Introduction}

The quantum properties characterizing hydrogen are fundamental constants important in many divergent areas of physics [1]. We focus on the electron, $\mathrm{e}^{-}$, Bohr radius, $a_{0}$, and the Rydberg constant, $R_{\infty}$, within hydrogen as a unified physical and mathematical system. These constants represent a mass, a distance, and a frequency (1/time) so they span the physical units. These constants are all known to be inter-related through power laws with the geometric factors of $2, \pi$, the fine structure constant, $\alpha$, the speed of light, $c$, and Planck's constant, $h$. In fact, each one of the hydrogen quantum constants is defined utilizing $\alpha, c, h$ in its derived unit value. None of them are experimentally directly measurable. This system is associated with all of the elements through Mosley's law. These quanta are related to the kinetic energy equation through Sommerfeld's original physical interpretation of $\alpha$ as a velocity divided by $c$, a dimensionless $\beta$. For the hydrogen system, the product of the rest mass of the electron times the speed of light times $\alpha$ both squared divided by 2 equals the hydrogen ionization energy. Through $\alpha$ and these constants, broad segments of physics are directly mathematically and conceptually inter-related within systematic power laws [2] [3].

Our goal is to evaluate these three quantum values as frequency equivalents: $v_{R}$, $v_{a_{0}}$, and $v_{e^{-}}$in the simplest fundamental manner, ratios, which are independent of any arbitrary physical unit system. We evaluate these three quantum constants all as natural unit frequency equivalents, $v \mathrm{~Hz}$ similar to Planck's time, and the Hartree atomic units [4]-[6]. This unit transformation is equivalent to Planck's constant, $h$, the speed of light, $c$, the electron charge, $e$, all scaled to 1 . By definition when $h$ equals 1 , its angular momentum is dimensionless. Their SI unit values can be reconstructed by multiplying the dimensionless calculation times the SI units. The final results are identical. We are searching for power law and harmonic sinusoidal patterns since these quanta are associated with quantum oscillators that have wave properties, and these constants are inter-related by many different power laws utilizing their SI units. Any other physical units such as mass, energy, or distance would result in the identical coupling constants, and not change the conclusions or dimensionless calculations. We conceptually choose frequency as our physical unit of choice since we are searching for wave properties.

We find that these three quantum ratios and $\alpha$ represents an integrated power law system broader than presently recognized. They are fundamentally related to the geometric factors of 2 and $\pi$ as a composite in $8 \pi^{2}$ [7]. It is possible to define any of these quantum values as a power law of any other quantum value utilizing these geometric factors. We also find that many other fundamental constants can be simply defined in terms of these three quanta in this dimensionless domain simplifying and unifying the unit system greatly.

\section{Methods}

All data for the constants' transformations to frequency equivalents were obtained from the websites: http://physics.nist.gov/cuu/Constants/, and http://physics.nist.gov/cuu/Constants/energy.html The NIST site has an online physical 
unit converter that can be used for these types of calculations. The respective frequency equivalents are: the Rydberg constant, $v_{R}, 3.28984196(17) \times 10^{15} \mathrm{~Hz}$, Bohr radius, $v_{a_{0}}$, $5.66525639(28) \times 10^{18} \mathrm{~Hz}$, and the electron, $v_{e^{-}}, 1.23558996(05) \times 10^{20} \mathrm{~Hz}$. The reciprocal of $\alpha$ is 137.0359999(78). The relative precision of the calculations is $5 \times 10^{-8}$, and limited by the uncertainty of $h$.

The italic number 1 is utilized after the standard constant's symbol to notate that is it scaled in the units of 1 for $h, c$, and $e$ rather than SI units, or in the dimensionless domain. Two examples are $\varepsilon_{0} 1$, and $h 1$. Many of the possible dimensionless and dimensional ratios of $v_{R}, v_{a_{0}}$, and $v_{e^{-}}$define fundamental integer power laws, and harmonic circle sinusoidal relationships.

We use a specialized notation to abbreviate the power law ratio relationships. These all represent fundamental constants. The notation allows for complicated ratios and powers to be expressed as text. Fundamental constants are commonly associated with super and subscript notations characterizing them. Capital $A$ is chosen to represent ratios. The superscript are the symbols for the frequencies of $v_{R}, v_{a_{0}}, v_{e^{-}}$or $\alpha$ of the numerator. The subscript is for the denominator. The powers of $v_{R}, v_{a_{0}}, v_{e^{-}}$or $\alpha$ are within parentheses to their right. These represent power laws so different points along a single power line are all inter-related. For example, $A_{v_{e}^{-(14)}}^{v_{a_{0}}(15)} H z, 1.028140195(51) H z$, and $A_{v_{a_{0}}}^{v_{e} e^{-(14)}} s, 0.972630001(49) s$ are reciprocals. All three of the following $A$ values fall on a common power law line, $A_{v_{e^{-}}(14)}^{v_{a_{0}}(15)} H z, \quad A_{e^{-}}^{v_{a_{0}}(15 / 14)} H z^{(1 / 14)} 1.00198421(05) H z^{(1 / 14)}$, and $A_{v_{e^{-}}^{v_{a_{0}}}}^{v_{14 / 15}} H z^{(1 / 15)}, 1.00185181(05) H Z^{(1 / 15)}$. Note that within the dimensional domain the $A$ powers related to $H z$ vary, but add to an integer power of $H z$ when transformed back to the SI units.

Equation (1a) demonstrates a pure geometric factor. $1 / 8 \pi^{2}$ equals the ratio of the product of $v_{e^{-}}$and $v_{R}$ divided by the square of $v_{a_{0}}$ [7]. This is the foundation of all of the other power laws. Different arrangements of equivalent relationships are shown as lettered Equations. Equation (1b) rearranges the equation to an identity Equation equaling 1 .

$$
\begin{aligned}
& \frac{1}{8 \pi^{2}}=\left(\frac{v_{e^{-}} v_{R}}{v_{a_{0}}^{2}}\right)=A_{v_{a_{0}}(2)}^{v^{e^{-} v_{R}}}=A_{v_{a_{0}}}^{v^{e}} A_{a_{0}}^{v_{R}} \\
& 1=8 \pi^{2}\left(\frac{v_{e^{-}} v_{R}}{v_{a_{0}}^{2}}\right)=8 \pi^{2} \frac{1}{2 \pi \alpha} \frac{\alpha}{4 \pi}=8 \pi^{2} A_{a_{0}}^{v_{e^{-}} v_{R}}=8 \pi^{2} A_{a_{0}}^{v_{R}} A_{v_{a_{0}}}^{v_{e^{-}}}
\end{aligned}
$$

Equations (2a), (2b), (3a, 3b), (4a), (4b), (4c) are all of the possible dimensionless frequency ratios of $v_{R} v_{a_{0}}$, and $v_{e^{-}}$; and are systematically related to $\alpha$ and the geometric factors: $2,2 \pi$, and $4 \pi$ [7]. Each represents another fundamental constant or relationship. $A_{e_{e^{-}}}^{v_{a_{0}}} 1$ is related to the ratio of $v_{a_{0}}$ divided by $v_{e^{-}}$, or $2 \pi \alpha$, and equals 0 . 0458506184(23). $A_{a_{0}}^{R} 1$ equals $v_{R}$ divided by $v_{a_{0}}, \alpha / 4 \pi$, and equals $5.80704866(29) \times$ $10^{-4}$. $A_{e^{-}}^{R} 1$ equals $v_{R}$ divided by $v_{e^{-}}, a^{2} / 2$, and equals $2.66256772(13) \times 10^{-5}$.

$$
\left(\frac{v_{a_{0}}}{2 \pi v_{e^{-}}}\right)=\alpha
$$




$$
\begin{aligned}
& \left(\frac{v_{a_{0}}}{v_{e^{-}}}\right)=2 \pi \alpha=A_{v_{e^{-}}}^{v_{a_{0}}} 1 \\
& \left(\frac{4 \pi v_{R}}{v_{a_{0}}}\right)=\alpha \\
& \left(\frac{v_{R}}{v_{a_{0}}}\right)=\frac{\alpha}{4 \pi}=A_{v_{a_{0}}}^{v_{R}} 1 \\
& \left(2 v_{R} / v_{e^{-}}\right)^{1 / 2}=\alpha \\
& \frac{v_{R}}{v_{e^{-}}}=\frac{\alpha^{2}}{2}=A_{v^{-}}^{v_{R}} 1 \\
& v_{R}=\frac{\alpha^{2}}{2} v_{e^{-}}=v_{e^{-}} A_{e^{-}}^{v_{R}} 1
\end{aligned}
$$

The derivation of the dimensionless values for permittivity, $\varepsilon_{0} 1$ and permeability, $\mu_{0} 1$, are shown in Equations (5)-(11). Here $\varepsilon_{0} 1$ equals $1 / \alpha, 137.0359999(78)$, and permeability, $\mu_{0} 1$ equals $\alpha, 7.29735256(36) \times 10^{-3}$. Equation (5) demonstrates the relationship between $\alpha$, electron charge, $e, \varepsilon_{0}, 2,4 \pi, h$, $\hbar$, and $c$ used in the derivation. Equation (6) derives the ionization energy of the electron in hydrogen. Equation (7) transforms the ionization energy from joules to frequency in $\mathrm{Hz}$ by dividing by $h$. In Equation (7) the $a_{0}$ is converted to a frequency equivalent utilizing a ratio with $c$. Equation (8) transforms the relationship to the dimensionless coupling constant of $v_{R}$ divided by $v_{a_{0}}$. Substitution of Equation (5) converts to the ratio of $\alpha$ and $4 \pi$, identical to Equation (3b).

$$
\begin{aligned}
& \alpha=\left(\frac{e^{2}}{2 \varepsilon_{0} h c}\right)=\frac{1}{4 \pi \varepsilon_{0}}\left(\frac{e^{2}}{\hbar c}\right)=\left(\frac{k_{e} e^{2}}{\hbar c}\right) \\
& E_{R}=\left(\frac{1}{4 \pi \varepsilon_{0}}\right)\left(\frac{e^{2}}{2 a_{0}}\right) \\
& v_{R}=\frac{E_{R}}{h}=\left(\frac{e^{2} v_{a_{0}}}{8 \pi \varepsilon_{0} h c}\right) \\
& \frac{v_{R}}{v_{a_{0}}}=\left(\frac{e^{2}}{8 \pi \varepsilon_{0} h c}\right)=\frac{1}{4 \pi}\left(\frac{e^{2}}{2 \varepsilon_{0} h c}\right)=\left(\frac{\alpha}{4 \pi}\right)=A_{v_{a_{0}}}^{v_{R}} 1=k_{e} 1
\end{aligned}
$$

Coulomb's dimensionless unit 1 constant, $k_{e} 1$, must equal $\alpha / 4 \pi, 5.80704866(29) \times$ $10^{-4}$. Equations (8) and (9) derive the dimensionless $\mathcal{E}_{0} 1$ constant from $k_{e} 1$. Here, $\mathcal{E}_{0} 1$ must equal $1 / \alpha, 137.0359999(78)$.

$$
k_{e} 1=\left(\frac{1}{4 \pi\left(\varepsilon_{0} 1\right)}\right)=\left(\frac{1}{4 \pi\left(\frac{1}{\alpha}\right)}\right)=\left(\frac{\alpha}{4 \pi}\right)=\frac{v_{R}}{v_{a_{0}}}=A_{a_{a_{0}}}^{\nu_{R}} 1
$$


The dimensionless relationship of $\varepsilon_{0} 1, \mu_{0} 1$, and $c 1$ is shown in Equation (10). $\mu_{0} 1$ equals $\alpha, 7.29735256(36) \times 10^{-3}$.

$$
\mu_{0} 1 \varepsilon_{0} 1 c 1^{2}=\alpha\left(\frac{1}{\alpha}\right) c 1^{2}=1
$$

The following is an example a power law derivation of one hydrogen quantum value from another utilizing a different, but parallel method. This example derives $v_{a_{0}}$ from $v_{R}, \alpha$, and $4 \pi$ based on Equation (3). Equation (11) evaluates the ratio of $4 \pi / \alpha$ or $A_{v_{R}}^{v_{a_{0}}}$ raised to a consecutive integer power series divided by $v_{R}$ in search of the value in seconds that is closest to 1 second. All of the constants demonstrate this power pattern. This is equivalent to when $4 \pi / \alpha$ or $A_{v_{R}}^{v_{a_{0}}}$ raised to a power is nearly equal to $V_{R} 1$. This can be substituted for a $v l$ value. The powers of $v_{a_{0}}$ and $v_{R}$ must be separated by 1 , so the ratio is not dimensionless. The $A$ values are related to identity relationship within these equations, but these $A$ values represent fundamental constants. These powers represent natural quantum number powers. They can be derived by searching arbitrarily through ratio power matrix of consecutive integer series for each constant's power. The ratio where the scalar is closest to 1 represents the $A$ ratio and the natural powers. Table 1 is an example of the type of arbitrary search for natural powers linking $v_{R}$ and $v_{a_{0}}$ All of the constants demonstrate this pattern with scalars near 1.

$$
\frac{\left(\frac{4 \pi}{\alpha}\right)^{n}}{v_{R}} S=\frac{\left(\frac{v_{a_{0}}}{v_{R}}\right)^{n}}{v_{R}} S=\left(\frac{v_{a_{0}}{ }^{n}}{v_{R}{ }^{n+1}}\right) S=A_{v_{R}(n+1)}^{v_{a_{0}}(n)} \approx 1 s
$$

For these two hydrogen constants, the $A_{v_{R}(n+1)}^{v_{a_{0}}(n)}$ value closest to 1s occurs when the $n$ power is 5 . The ratio of $4 \pi / \alpha$ to the fifth power, equals $v_{R}$ times $v_{a_{0}}^{5}$ divided by $v_{R}^{6}$, or the product of $v_{R}$ and $A_{v_{R}(6)}^{v_{a_{0}}(5)} s$, Equation (12). Here, $4 \pi / \alpha$ to the fifth power equal $1.514337482(75) \times 10^{16}$.

$$
\left(\frac{4 \pi}{\alpha}\right)^{5}=v_{R} H z\left(\frac{v_{a_{0}}^{5}}{v_{R}^{6}}\right) s=v_{R} H z A_{v_{R}(6)}^{v_{a_{0}}(5)} s=v_{R} H z \times 4.60307060(23) \mathrm{s}
$$

Table 1. Power law ratio matrix.

\begin{tabular}{ccccc}
\hline$v_{R}^{\text {row }} / v_{a_{0}}^{\text {column }}$ & 4 & 5 & 6 & 7 \\
\hline 4 & $1.13 \times 10^{-13}$ & $2.00 \times 10^{-32} s$ & $3.54 \times 10^{-51} s^{2}$ & $6.25 \times 10^{-70} s^{3}$ \\
5 & $3.74 \times 10^{2} \mathrm{~Hz}$ & $6.60 \times 10^{-17}$ & $1.16 \times 10^{-35} \mathrm{~s}$ & $2.05 \times 10^{-54} \mathrm{~s}^{2}$ \\
6 & $1.23 \times 10^{18} \mathrm{HZ}^{2}$ & $2.17 \times 10^{-1} \mathrm{~Hz}$ & $3.83 \times 10^{-20}$ & $6.76 \times 10^{-39} \mathrm{~s}$ \\
7 & $4.04 \times 10^{33} \mathrm{~Hz}^{3}$ & $7.14 \times 10^{14} \mathrm{~Hz}^{14}$ & $1.26 \times 10^{-4} \mathrm{~Hz}$ & $2.22 \times 10^{-23}$ \\
\hline
\end{tabular}

Table 1 is a power ratio matrix of $v_{R}$ raised to the row power divided by $v_{a 0}$ raised to the column power where the powers are consecutive integer series. The ratio with a scalar value closest to 1 is searched for within an arbitrary matrix. Those powers represent the natural quantum number powers linking those two constants. This occurs with $V_{R}$ raised to the $6^{\text {th }}$ power divided by $V_{\text {an }}$ raised to the $5^{\text {th }}$ power. The ratio is $2.17 \times 10^{-1} \mathrm{~Hz}$. Note that all of the other values are widely divergent from 1 . Here $2.17 \times 10^{-1} \mathrm{~Hz}$ is the reciprocal of $A_{v_{R}(6)}^{v_{0}(5)}$, and the reciprocal of $4.06 s$ as seen in Equations (12)-(15). 
The ratio relationships in Equations (2)-(4) are solved for any hydrogen quantum value, and are raised to the ninth power which is closest to a scalar value of 1 , for this example $v_{a_{0}}$, and the power of 5 . This exposes the powers of $\mathrm{Hz}$ and seconds, Equation (13). The derived value for $4 \pi / \alpha$ raised to the fifth power is substituted from Equation (12) into Equation (13). The fifth power of $v_{a_{0}}$ is derived from the product of $v_{R}$ raised to the sixth power and $A_{v_{R}(6)}^{v_{a_{0}}(5)} s$.

$$
v_{a_{0}}^{5}=\left(\frac{4 \pi}{\alpha}\right)^{5} v_{R}^{5}=v_{R}^{6} H z^{6}\left(\frac{v_{a_{0}}^{5}}{v_{R}^{5}}\right) s=v_{R}^{6} H z^{6} A_{v_{R}(6)}^{v_{a_{0}}(5)} S
$$

In Equation (14) $v_{a_{0}}$ is derived from fractional powers of $v_{R}$. Here, $A_{v_{R}(6 / 5)}^{v_{a_{0}}} s^{(1 / 5)}$ equals $1.35709274(07) \mathrm{s}^{(1 / 5)}$.

$$
v_{a_{0}}=\left(\frac{4 \pi}{\alpha}\right) v_{R}=v_{R}^{6 / 5} H z^{6 / 5}\left(\frac{v_{a_{0}}}{v_{R}^{6 / 5}}\right) s^{(1 / 5)}=v_{R}^{6 / 5} H z^{6 / 5} A_{v_{R}(6 / 5)}^{v_{a_{0}}} s^{(1 / 5)}
$$

Equation (15) is another power law variation of this relationship deriving $v_{a_{0}}$ from $v_{R}$. $A_{v_{a_{0}}(5)}^{v_{R}(6)} \mathrm{Hz}$ equals $0.21724628739519 \mathrm{~Hz}$. The sixth power of $4 \pi / \alpha$ equals $2.60775752(14) \times 10^{19}$.

$$
v_{a_{0}}=\left(\frac{(4 \pi)^{6}}{\alpha^{6}}\right)\left(\frac{\left(v_{R}\right)^{6}}{\left(v_{a_{0}}\right)^{5}}\right) H z=\left(\frac{(4 \pi)^{6}}{\alpha^{6}}\right) A_{a_{0}(5)}^{R(6)} H z
$$

Equation (16) is another example that derives $v_{e^{-}}$from $v_{R}$. Here, $A_{v_{R}(9 / 7)}^{v_{e}^{-}} s^{(2 / 7)}$ equals $1.38426983(05) \mathrm{s}^{(2 / 7)}$.

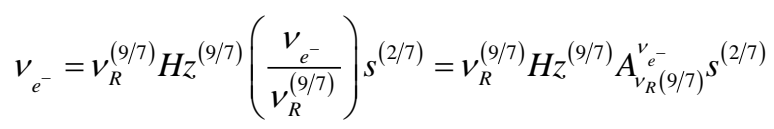

\section{Results}

Equation (1) is related to Planck's constant in the dimensionless domain, and equals 1, Equations (17a), (17b). This is logical since $h 1$ is intentionally scaled as 1 in this system since energy and frequency are scaled identically in a pure frequency domain. This ratio is equivalent to an angular momentum since the ratio represents the product of a mass, a frequency, a distance squared, $\left(\mathrm{kg} \cdot \mathrm{m}^{2} / \mathrm{s}\right)$ in the SI dimensional domain. Since this ratio is in the frequency domain, a distance is related to the reciprocal of the frequency equivalent. This relationship is related to the annihilation energy of a mass. Equation (17b) demonstrates that the reduced Planck's constant, $\hbar$, equals $1 / 2$ in the dimensionless domain, $\hbar 1$.

$$
\begin{aligned}
& h 1=8 \pi^{2}\left(\frac{v_{e^{-}} v_{R}}{v_{a_{0}}^{2}}\right)=8 \pi^{2} A_{v_{a_{0}}(2)}^{v_{e^{-}}^{v^{-}}}=8 \pi^{2} \frac{1}{2 \pi \alpha} \frac{\alpha}{4 \pi}=8 \pi^{2} A_{v_{a_{0}}}^{v_{R}} A_{v_{a_{0}}}^{v_{e^{-}}}=1 \\
& \hbar 1=4 \pi\left(\frac{\left(v_{R} s\right)\left(v_{e^{-}} s\right)}{\left(v_{a_{0}}\right)^{2}}\right)=\frac{1}{2}
\end{aligned}
$$


Equations (18a) and (18b) demonstrate that this same relationship is valid in the standard SI unit equations of $h$ and $\hbar$.

$$
\begin{aligned}
& h=8 \pi^{2} m_{e^{-}} v_{R} a_{0}^{2} \\
& \hbar=4 \pi m_{e^{-}} v_{R} a_{0}^{2}
\end{aligned}
$$

Equation (4c) is equivalent to the prototype kinetic energy equation where the product of a mass times the velocity divided by $c, \beta$ squared divided by 2 .

Equation (2) demonstrates that the $\alpha$ derivation from $v_{a_{0}}$ and $v_{e^{-}}$is within a circle equation related to a radius, circumference, and $2 \pi$. This must be related to a sinusoidal harmonic system, and a fundamental momentum relationship.

The dimensionless constants that define $\varepsilon_{0} 1, \mu_{0} 1$, and $k_{e} 1$ are all very simple values related to $\alpha$ and geometric factors, Equations (8)-(10). $k_{e} 1$ logically is related to $\alpha /(4 \pi)$ since the energy is proportional to the distance frequency equivalent. This is a universal relationship for any distance as a frequency equivalent. Here, $v_{a_{0}}$ times $\alpha /(4 \pi)$ equals $v_{R}$, Equation (3).

Equations (12)-(16) demonstrate that if any two of the quantum values, including an $A$ factor, are known then all of the constants can be derived.

The $A$ factors do not represent errors, but are fundamental essential constants incorporating the geometric factors that are imbedded within the quantum domain scalar values. These geometric factors represent conversion factors that bridge between Euclid geometry; and the power laws of $v_{R}, v_{a_{0}}, v_{e^{-}}$and $\alpha$. The geometric factors are essential in projecting the power laws into the harmonic linear domain, and three dimensional spaces from a system solely defined by sinusoidal waves. Here, 2 is related to kinetic energy, Equation (4). In Equations (17a) and (17b), the factor 2 is similar to the 2 in the Schwarzschild equation since it refers to the transition of matter to an annihilation boson state. This is not a kinetic state. Here, $2 \pi$ is related to a radius and its associated circumference as seen in Equations (17a) and (17b). Here, $4 \pi$ is related to $4 \pi$ times the radius squared that defines the surface of the sphere seen in force fields such as electrical charges, or magnetic fields, as in $k_{e} 1$, Equations (3), (5), (7)-(9), (11)-(15).

\section{Discussion}

The existing physical unit system of SI units is an arbitrary system of mass, time, distance, and energy units. They were chosen for measurement convenience, and their previous utilization/standardization. In quantum physics integer quantum numbers are associated with natural quantum units, such as the Rydberg constant. Planck's time is a classic time natural unit. Quantum spin is associated with $h$ as a natural unit. This method has a long history, and is a valid approach in physics [4]-[6]. Natural units are not arbitrary, and essential if the system is to be evaluated from a quantum number perspective. The ratio of two natural units of a single physical unit represents a fundamental dimensionless scaling ratio. The Standard Model is not helpful in most scaling relationships between particles. The Higgs boson could not be predicted with accuracy within the Standard Model. The Buckingham Pi theory of dimensionless analysis of 
physical systems is well established, and states that physical systems can be analyzed in a dimensionless domain. These reasons are the rationale for the methods utilized to search for the most fundamental quantum relationships.

This paper demonstrates that many of the fundamental constants in this hydrogen system demonstrate very simple, and integrate definitions as ratios of three hydrogen quanta and geometric factors. It is logical that all of these ratios should be related to fundamental constants and relationships. These are truly the only three constants in this global power law system. This is not the typical concept of these individual constants. Though they are inter-related they are not thought of as being only ratio or product projections of just three quanta. Equations (1), (17a), (17b) demonstrate that Planck's constant is related to the dimensionless ratio of the frequencies defining an angular momentum scaled to the number 1 . This is anticipated in a natural unit system. Planck's reduced, $\hbar 1$, constant is scaled to $1 / 2$. This is logical since Planck's constant times one $\mathrm{Hz}$ represents at minimum unit of energy per cycle. This equates the annihilation energy of a mass to an equivalent frequency. The $h 1$ is directly related to a spin of 1 since it refers to $1 \mathrm{~Hz}$ times a dimensionless value associated with the energy of a wave with a frequency of $1 \mathrm{~Hz}$. Photons have a spin of 1 . The reduced Planck's constant $\hbar 1$ is $1 / 2$, and associated with a spin of $1 / 2$ of a fermion, in this case the electron. The $8 \pi^{2}$ geometric factor is the composite of $2 \pi$ squared, $4 \pi^{2}$; and 2 . The $2 \pi$ squared scales the circumference distance an electron orbiting the proton. The 2 is related to the fact this angular momentum refers to the process of mass annihilation to photons, not to a kinetic energy. $8 \pi^{2}$ is also found as the geometric factor of the Schrodinger equation.

Multiple different ratios of $v_{R}, v_{a_{0}}, v_{e^{-}}$, and geometric factors are related to $\alpha$, Equations (1)-(4). One of them is equivalent to the kinetic energy of a mass as a prototype form for all kinetic energies. One of the ratios is related to $2 \pi$ a relationship of $v_{a_{0}}$ and $v_{e^{-}}$, Equation (2). This demonstrates a harmonic sinusoidal nature to this system. This must also define a fundamental relationship between distance and mass, (Equation (2)) similar to that seen with distance and energy in Equation (3). This must be related to a linear momentum. The Coulomb constant's dimensionless value, $k_{e} 1$ is related to the ratio of $v_{R}$ divided by $v_{a_{0}}$, or $\alpha / 4 \pi$. Here $4 \pi$ is the classic geometric factor related to a spherical surface and a force field. Dimensionless permittivity and permeability equal $1 / \alpha$, and $\alpha$. This is a remarkably unified and simplified system of units demonstrating their most fundamental scaling inter-relationships.

This work demonstrates that there are a myriad of power law relationships where any constant can derived if any two are known including an $A$ value, Equations (12)-(16). There are many power laws of these constants in the SI unit domain, but since their scaling relationships are not apparent these types of derivations starting with only three quanta are not possible. We present only a few of the possible power laws. In the SI unit system there are many different constants that appear to be unique values, but based on this analysis they actually represent only a product or ratio combination of the same three hydrogen quanta. Equation (5) is a standard unit equation and inter-relates five different physical constants. In this dimensionless unified domain three of the con- 
stants drop out from the scaling of the calculations as 1's. The other two are inter-related by a geometric factor. Two of the constants are both related to the same natural unit value, $\alpha$. There is really only one essential quantum constant that inter-relates all five of the SI values.

These power law relationships are not felt to coincidental or mathematically contrived, but rather fundamental. The ratio of the $v_{a_{0}}$ to the 15 power divided by $v_{e^{-}}$ to the 14 power equals $1.028140195(51) \mathrm{Hz}$. This is a ratio of $1.987438 \times 10^{281} \mathrm{~Hz}^{15} \mathrm{di}$ vided by $1.933042 \times 10^{281} \mathrm{~Hz}^{14}$. It is highly unlikely that ratios of the integer powers of these quantum values, that represent gigantic scalar values far beyond what is typical, are all nearly equal to 1 by chance. Every hydrogen quantum constant demonstrates a similar pattern. It is also essential that they not be equal to 1 so that is not a logic or mathematical error. They cannot be equal to 1 since the geometric factors are imbedded in their scalar values. There are many similar fundamental ratio power laws, and most are not known. A recent paper describes the quantum mechanical derivation of the Wallis formula for $\pi$ [8]. Therefore, these types of relationships between the quantum constants and geometric factors of 2 and $\pi$ do not represent inappropriate speculation, but are logically imbedded within the constants' natural unit scaling.

\section{Conclusion}

This paper describes the fundamental scaling relationships between the unified physical system of the electron, Bohr radius, and Rydberg constant of hydrogen within a natural unit dimensionless or dimensional system. It is found that classic geometric factors are embedded within the scaling of these quanta. This system bridges from the quantum wave domain to three dimensional space domains. When the relationships of these three constants are analyzed, their simple ratios and products project out to a wide array of other fundamental constants and relationships. There are also a myriad of power laws, and $A$ values, some known and some new, inter-relating these constants so that they can all be derived from the knowledge of just two. These finding demonstrate that the fundamental constants of hydrogen represent a highly integrated logical harmonic power law system that extends beyond hydrogen. This represents a new perspective on the Standard Model within a parallel power law system.

\section{Acknowledgements}

I would like to thank Richard White M. D., Vola Andrianarijaon PhD, Keshab Sapkota $\mathrm{PhD}$, and Richard Vento MS for their help.

\section{References}

[1] Zhao P., Lichten, W. and Zhou, Z. (1989) Physical Review A, 29, 2888. http://dx.doi.org/10.1103/PhysRevA.39.2888

[2] Saichev, A., Malevergne, Y. and Sornette, D. (2009) Lecture Notes in Economics and Mathematical Systems, 632.

[3] Katznelson, Y. (2004) An Introduction to Harmonic Analysis. 3rd Edition, Cambridge 
University Press, Cambridge. http://dx.doi.org/10.1017/CBO9781139165372

[4] Mills, J. (1932) The Journal of Physical Chemistry, 36, 1089-1107. http://dx.doi.org/10.1021/j150334a001

[5] Karshenboima, S. (2008) The European Physical Journal Special Topics, 163, 141.

[6] Flowers, J. and Petley, B. (2001) Reports on Progress in Physics, 64, 1191. http://dx.doi.org/10.1088/0034-4885/64/10/201

[7] Chakeres, D. (2011) Particle Physics Insights, 4, 33. http://dx.doi.org/10.4137/PPI.S8269

[8] Friedmann, T. and Hagen, C. (2015) Journal of Mathematical Physics, 56, 112101. http://dx.doi.org/10.1063/1.4930800

\section{Submit or recommend next manuscript to SCIRP and we will provide best service} for you:

Accepting pre-submission inquiries through Email, Facebook, LinkedIn, Twitter, etc. A wide selection of journals (inclusive of 9 subjects, more than 200 journals)

Providing 24-hour high-quality service

User-friendly online submission system

Fair and swift peer-review system

Efficient typesetting and proofreading procedure

Display of the result of downloads and visits, as well as the number of cited articles

Maximum dissemination of your research work

Submit your manuscript at: http://papersubmission.scirp.org/

Or contact jmp@scirp.org 